\title{
Simultaneous interplanetary scintillation and optical measurements of the acceleration of the slow solar wind
}

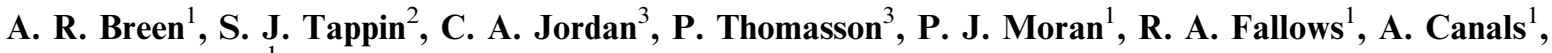 \\ P. J. S. Williams ${ }^{1}$ \\ ${ }^{1}$ University of Wales, Aberystwyth, Ceredigion SY23 3BZ, Wales, UK \\ ${ }^{2}$ University of Birmingham, Edgbaston, Birmingham, UK \\ ${ }^{3}$ The University of Manchester, Jodrell Bank Observatory, Cheshire, UK
}

Received: 21 February 2000 / Revised: 13 June 2000 / Accepted: 21 June 2000

\begin{abstract}
Simultaneous observations of the slow solar wind off the southeast limb of the Sun were made in May 1999 using optical measurements from the C2 and C3 LASCO coronagraphs on board the SOHO spacecraft and radio-scattering measurements from the MERLIN and EISCAT facilities. The observations show the slow solar wind accelerating outwards from 4.5 solar radii (R), reaching a final velocity of $200-300 \mathrm{~km} \mathrm{~s}^{-1}$ by 25 $30 \mathrm{R}$. The acceleration profile indicated by these results is more gentle than the average profile seen in earlier LASCO observations of larger scale features, but is within the variation seen in these studies.
\end{abstract}

Key words: Interplanetary physics (solar wind plasma; sources of the solar wind; instruments and techniques)

\section{Introduction}

\subsection{Interplanetary scintillation observations}

Observations of interplanetary scintillation (IPS), in which the diffraction pattern cast across the Earth when a distant compact radio source is observed through the solar wind have been used as a flow tracer in the solar wind for over 35 years (e.g. Hewish et al., 1964; Dennison and Hewish, 1967; Armstrong and Coles, 1972). Provided that the phase changes introduced into the incident radio waves are small (the condition of weak scattering) it is possible to treat contributions to the observed scintillation pattern from different parts of the line-of-sight to the source separately (e.g. Coles, 1995; Grall et al., 1996; Breen et al., 1996b). As the raypath from the source to the antenna moves closer in to the Sun, the phase changes become larger and the amplitude of the scintillation pattern increases until the

Correspondence to: A. R. Breen

e-mail: azb@aber.ac.uk inner limit of weak scattering is reached. Inside this limit, in the "strong scattering" regime where the phase changes introduced by scattering are large, the contributions from different regions of the ray-path do not combine as a simple weighted linear sum, and it becomes very difficult to accurately determine the contributions of different regions of the ray-path to the observed scintillation pattern (e.g. Hewish, 1989; Grall, 1995). The distance from the Sun for the transition from weak to strong scattering depends on the frequency of the radio waves received: for EISCAT observing at $0.93 \mathrm{GHz}$, the transition takes place at 25-30 solar radii (R) in the slow wind; for MERLIN (Thomasson, 1986) observing at $5 \mathrm{GHz}$, the transition lies below $7 \mathrm{R}$, though it is to be noted that the power radiated by most of the strong astronomical radio sources falls off towards higher frequencies, so that fewer "good" IPS sources are available at higher frequencies. A combination of high-frequency observations covering the region close to the Sun and lower-frequency observations covering the outer regions, with overlapping fields-ofview, is ideal. A schematic diagram of the geometry of an IPS observation is given in Fig. 1.

The first IPS observations using the EISCAT facility at a frequency of $0.93 \mathrm{GHz}$ were made in 1982 (Bourgois et al., 1985) and a regular series of measurements of solar wind velocity have been made every summer since 1993 . IPS observations have been made with MERLIN by Rickett (1992), initially in 1989 at $1.658 \mathrm{GHz}$ using several baselines up to $127 \mathrm{~km}$ in length, and later in $1992 \mathrm{using}$ the upgraded MERLIN incorporating the new Cambridge antenna which provides baselines of up to $217 \mathrm{~km}$. The results of these observations were encouraging, and provided the impetus for the combined programme of EISCAT and MERLIN observations run in May 1999.

\subsection{White-light observations}

One of the aims of the coronal instruments on the SOHO spacecraft was the determination of the acceleration profile of the solar wind (Domingo et al., 1995). 


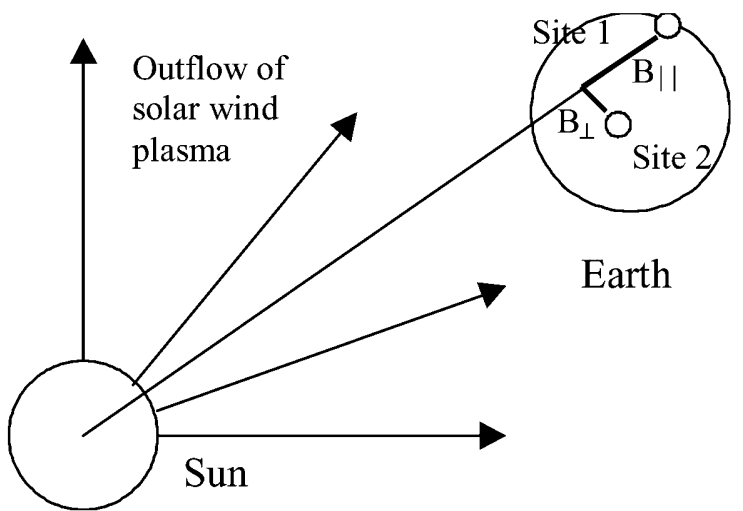

Fig. 1. Schematic diagram of a two-station observation of interplanetary scintillation, viewed from the direction of the radio source (normally a Quasar). The maximum cross-correlation between the scintillations observed at the two sites occurs when the baseline between the two receiving stations is parallel to the weighted average direction of flow of the solar wind across the ray-path (close to radially outwards from the Sun), and at a time-lag determined by the drift velocity of the scintillation pattern between the two ray-paths (e.g. Moran et al., 1998). The baselines radial and tangential to the Sun-Earth direction in the plane of the sky are shown as $B_{\|}$and $B_{\perp}$ respectively

Of the imaging coronal instruments, the large-angle spectroscopic coronagraph (LASCO, described by Brueckner et al., 1995) is ideal for investigating outflow velocities from the inner corona out to distances of 25 30 R. Various LASCO studies (Sheeley et al., 1997; Tappin and Simnett, 1998; Tappin et al., 1999) have investigated the speed of identifiable features as they propagated out through the fields-of-view of the two LASCO coronagraphs. The small discrete "knots" or "blobs" studied by Sheeley et al. (1997) showed evidence of continuous acceleration from around 2 solar radii $(\mathrm{R})$ out to beyond $20 \mathrm{R}$. The large-scale features, coronal mass ejections, studied by Tappin and Simnett (1998) were somewhat different. Some showed continuous acceleration, but the majority either moved at constant velocity or underwent a sudden acceleration at distances between 5 and $8 \mathrm{R}$. This behaviour is similar to that reported by Srivastava et al. (1999).

Sheeley et al. (1997) derived flow speeds by assuming that the features they studied were drifting at the background solar wind speed "like leaves in a stream". This assumption can be made with more certainty if smaller features are studied, and particularly if the velocity profiles of features on different scales are compared. Tappin et al. (1999) considered very smallscale features in LASCO data and derived outflow velocities by calculating the cross-correlation function of white-light intensity at two radial distances from the limb of the Sun, a method analogous to the 2-site IPS technique (but see Tappin et al., 1999 for a discussion of the differences between the two methods). The results indicated that the slow solar wind had reached its cruising speed by 25-30 R, with most of the acceleration taking place inside 10-15 R. Breen et al. (2000) compared the velocity of small-scale variations in intensity observed in the fast solar wind with velocities obtained from EISCAT IPS measurements, but unfortunately the signal-to-noise ratio in the outer part of the LASCO field-of-view was too low for a direct comparison of velocities derived by the two techniques at the same radial distance. The observations made by Tappin and Simnett (1998), Sheeley et al. (1997), Tappin et al. (1999), Srivastava et al. (1999) and Breen et al. (2000) therefore cover four entirely different ranges of irregularity scale, from in excess of $100000 \mathrm{~km}$ to around $100 \mathrm{~km}$. In this study LASCO, MERLIN and EISCAT data from overlapping fields-of-view were compared for three days in May 1999. The scale sizes of the irregularities ranged from $\sim 10000 \mathrm{~km}$ for the LASCO features to $\sim 100 \mathrm{~km}$ for the irregularities observed by EISCAT IPS and $\sim 60 \mathrm{~km}$ for those observed at $5 \mathrm{GHz}$ by MERLIN.

\section{Observations}

The observations discussed were made from EISCAT, MERLIN and LASCO between 2 and 15 May, 1999 and were co-ordinated so that the different instruments observed flow along similar stream lines of flow (within $\pm 10^{\circ}$ latitude and lying off the same limb).

\subsection{LASCO observations}

The LASCO (Large-Angle Spectroscopic Coronagraph) instruments observed the white-light intensity in the solar corona off the southeast limb of the Sun for $12 \mathrm{~h}$ per day on 9 and 11 May, 1999, producing an image from the $\mathrm{C} 2$ and $\mathrm{C} 3$ coronagraphs every $12 \mathrm{~min}$. This was the finest time resolution possible when using $\mathrm{C} 2$ and $\mathrm{C} 3$ together, because of limitations in the data transfer rate from SOHO to Earth (Plunkett, private communication, 1999). The results were divided into two overlapping distance ranges from the Sun (Fig. 2) and cross-correlated (using the analysis technique described by Tappin et al., 1999) to obtain estimates of the flow speed. The observations from each day were divided into two 6-h intervals to provide independent estimates of slow speed.

\subsection{MERLIN observations}

The strong radio source $0318+164$ (CTA-21), with its small angular size and well-studied structure, is an ideal source for high-frequency IPS observations of the innermost regions of the solar wind. Lying off the southeast limb of the Sun in early May 1999 and moving closer to the Sun and towards higher latitudes with time, it had ray-paths to the Earth which reached their closest approach to the Sun on 12 May, 1999.

Time was allocated for MERLIN observations on three days (4, 9 and 11 May, 1999). 0318+164 was observed on all three days for approximately 2.5 hours per day. The observations are summarised in Table 1. 


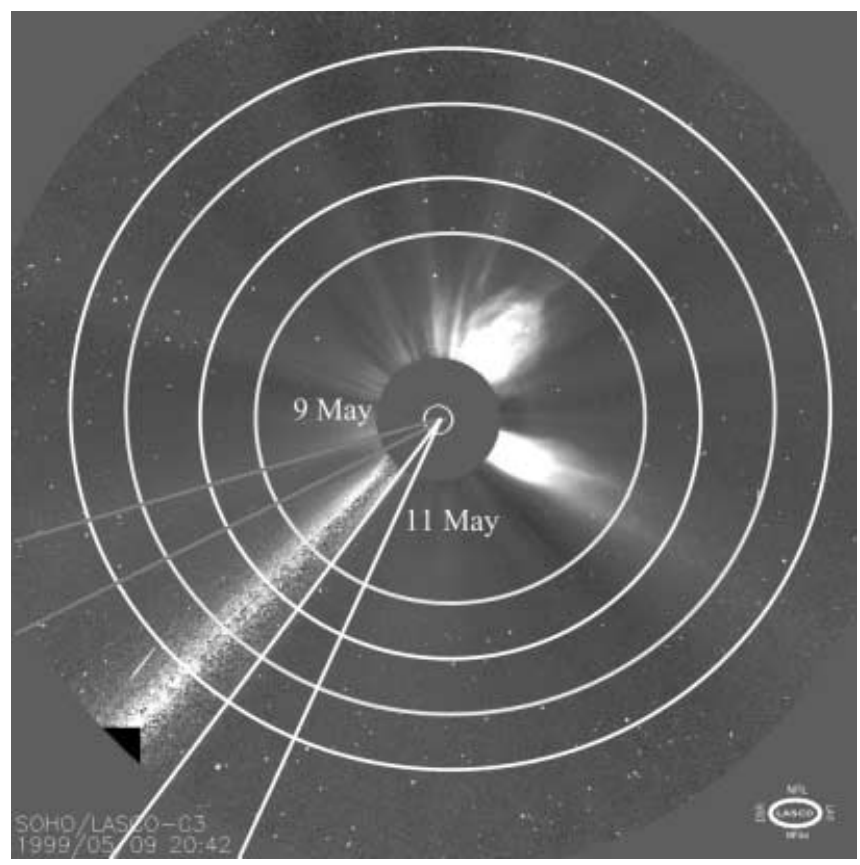

Fig. 2. Schematic diagram showing the regions of the LASCO fieldof-view used to estimate flow speeds on 9 and 11 May, 1999, overlaid on a LASCO C3 image from 2042 UT on 9 May, 1999. The support for the occulting disc is visible at about $45^{\circ}$ South off the east limb. The regions observed were PA 110-125 on 9 May and PA 145-160 on 11 May

Observations were also made of a second radio source, $0321+123$, which lay further out from the Sun off the same limb as $0318+164$, but unfortunately these proved to be too weak to produce useful scintillation measurements. 3C84 $(0316+413)$ and $0552+398$, at sufficiently large distances from the Sun that the degree of scintillation should have been negligible were observed for calibration purposes.

The observations on 4 May were made using the Cambridge and Knockin telescopes of MERLIN, operating at a central frequency of $1.66 \mathrm{GHz}$, a single polarisation (left circular) of equivalent band width $10 \mathrm{MHz}$ being sampled at $100 \mathrm{~Hz}$. On 9 and $11 \mathrm{May}$, the Cambridge and Jodrell Bank Mk2 telescopes were used at a central frequency of $5 \mathrm{GHz}$. Once again the detected equivalent band width was $10 \mathrm{MHz}$ and the signals were sampled at $100 \mathrm{~Hz}$. However, although only the left-circular polarisation was recorded from the Cambridge telescope, both left- and right-circular polarisations were recorded from the Mk2 telescope Ideally, both polarisations would have been recorded at all sites. However, in order to preserve the total power information essential for IPS measurements during the microwave link signal transfer from the outstation sites at Cambridge and Knockin to Jodrell Bank the signals had to be frequency modulated onto the links, which restricted them to a single $10 \mathrm{MHz}$ polarisation band. In future experiments we intend to record data locally at each site, which will permit a wider band width and sampling of both polarisations.

Although the Cambridge data were noisy, with significant quasi-periodic interference, the scintillation pattern as the solar wind flowed across the ray-path from $0318+164$ was detectable and good correlation was observed between the measurements at the two sites (Fig. 3). Good data were obtained from Knockin on 4 May, 1999, and from the Jodrell Bank Mk.2 telescope on 9 and 11 May, 1999.

The baselines available from MERLIN are significantly shorter than those of EISCAT (Tables 1, 2), but the velocity resolution is still adequate when the solar wind across the ray-path is dominated by a single stream, as was the case for the observations of 0318+164 during early May 1999 .

\subsection{EISCAT observations}

EISCAT made observations of IPS off the southeast limb of the Sun every day from 2 May, 1999, to 15 May, 1999. These observations are summarised in Table 2. The data were received on one polarisation over a $8 \mathrm{MHz}$ band width at the Tromsø and Sodankylä sites (7.5 MHz at the Kiruna site), centred on $931.5 \mathrm{MHz}$. Initial sampling was at $10 \mathrm{kHz}$, with the data subsequently averaged to give a sample every $0.01 \mathrm{~s}$ (e.g. Breen et al., 1996a). Together with the MERLIN and LASCO observations, the EISCAT results extended the measured profile of slow wind velocity out to beyond $90 \mathrm{R}$. Mean velocities throughout the distance range covered by EISCAT were in the range $250-380 \mathrm{~km} \mathrm{~s}^{-1}$.

\section{Results}

The LASCO results were dominated by slow flow throughout this period, though a small amount of fast flow was detectable. The IPS results from MERLIN and EISCAT also clearly showed clear slow flow with a weak fast component originating from a coronal hole lying on the anti-Earthward side of the Sun. This was as expected, as the observations lay above a conspicuous
Table 1. MERLIN IPS observations during the slow wind velocity study interval. Latitudes given are heliocentric, longitudes are Carrington and distances are in solar radii: these co-ordinates are given for the point of closest approach of the IPS ray-path to the Sun. Baseline lengths are in $\mathrm{km}$, velocities are in $\mathrm{km} \mathrm{s}^{-1}$

\begin{tabular}{lllllllll}
\hline Date & Source & Latitude & Longitude & Distance & Baseline $_{\|}$ & Baseline $_{\perp}$ & Velocity $^{\Delta V e l o c i t y}$ \\
\hline 990504 & $0318+164$ & -19 & 242 & 30.8 & 152 & 0.8 & 316 & 25 \\
990509 & $0318+164$ & -36 & 166 & 13.6 & 154 & 0.2 & 169 & 84 \\
990511 & $0318+164$ & -60 & 138 & 8.4 & 156 & 0.2 & 105 & 26 \\
\hline
\end{tabular}


$\mathrm{s}=$ start $\mathrm{f}=$ end $\mathrm{x}=$ delete $\mathrm{q}=$ done $\mathrm{r}=$ reject $\mathrm{c}=$ threshold clip $\mathrm{p}=$ replot $0318+164$ ON 990509 AT 151027 BOT-TOP JMK2 CAMB
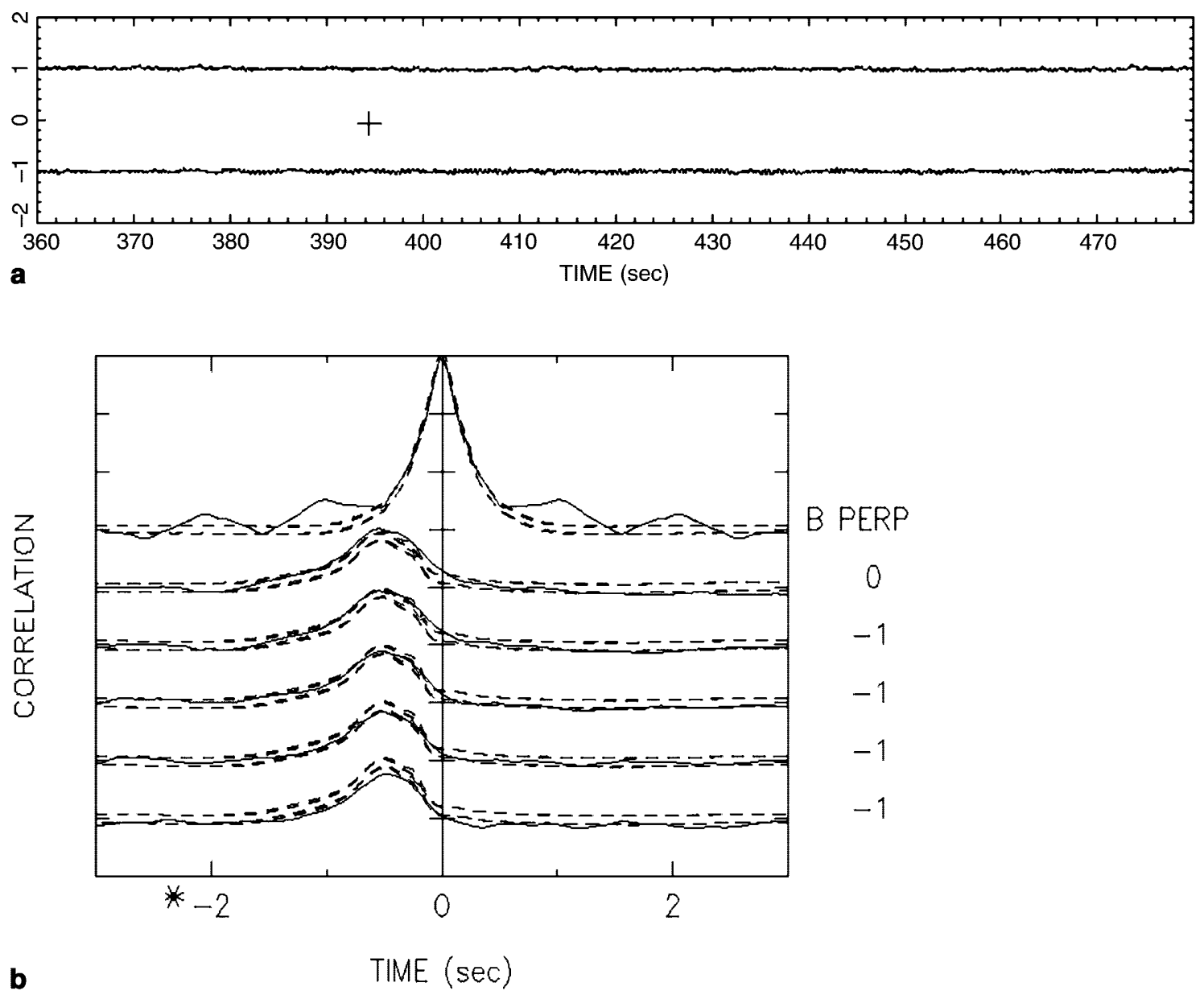

Fig. 3. a Raw data from the MERLIN receiving stations at Jodrell Bank (average of scintillations in both polarisations) and Cambridge, 9 May, 19991507 UT to 1509 UT. b Observed (solid lines) and fitted auto- and cross-correlation functions for $75 \mathrm{~min}$ of data centred on

east-limb streamer (Fig. 2). Projecting the IPS ray-paths back to $2.5 \mathrm{R}$ using a simple constant-velocity ballistic model shows that the great majority of flow across the ray-paths originated above the bright corona. A typical example (MERLIN observation of $0318+164$ on 9 May, 1999) is shown as Fig. 4.

The LASCO velocities are shown in Fig. 5. The large error bars at heliocentric distances beyond $25 \mathrm{R}$ are a result of the poor signal-to-noise ratio in the outer regions of the LASCO field-of view, which are caused by the low electron densities (and thus poor contrast between the white-light corona and the background) at these distances.

Figure 6 shows the velocities derived from MERLIN and EISCAT IPS observations on 4, 9 and 11 May, 1999. There is considerable scatter between data points, which is probably a result of different streams of slow wind lying across the ray-paths of the different observations, but the overall trend, with acceleration from velocities of $100-200 \mathrm{~km} \mathrm{~s}^{-1}$ at distances of $9-11 \mathrm{R}$ to 250 $300 \mathrm{~km} \mathrm{~s}^{-1}$ by $30 \mathrm{R}$, is clear. The velocities observed on 4 May, 1999, at $31 \mathrm{R}$ by MERLIN (at $1.66 \mathrm{GHz}$ ) and
1540 UT. The observed scintillation is weak but clearly detectable, and model fitting suggests that the data is best described as dominated by slow flow at an average speed of $160 \mathrm{~km} \mathrm{~s}^{-1}$, with a significant degree of variation $\left( \pm 100 \mathrm{~km} \mathrm{~s}^{-1}\right)$

EISCAT (at $931.5 \mathrm{MHz}$ ) agree to within the uncertainties in the measurements. There is good reason to believe that a significant proportion of these uncertainties reflect real, physical differences in the velocities of different streams of slow wind across the ray-paths.

\section{Slow wind velocity profiles}

Figure 7 shows a plot of the velocities derived from all of the observations made by LASCO, MERLIN and EISCAT between 2 and 15 May, 1999. There is very good overall agreement between the LASCO and IPS velocities, indicating that the irregularities observed by the two techniques are drifting at the same speed. As the scale sizes of the irregularities observed by LASCO and IPS are so different $(\sim 10000 \mathrm{~km}$ for LASCO, $\sim 100 \mathrm{~km}$ for IPS) this suggests that the irregularities are drifting at the background flow speed.

The observations made on 4, 9 and 11 May covered different latitudes and longitudes on the Sun, so any direct comparison of results from different distances on 
Table 2. EISCAT IPS observations during the slow wind velocity study interval. Latitudes given are heliocentric, longitudes are Carrington and distances are in solar radii: these co-ordinates are given for the point of closest approach of the IPS ray-path to the Sun. Velocities are in $\mathrm{km} \mathrm{s}^{-1}$. Observations shown in normal type lie along similar stream lines of flow to that observed using MERLIN on 4 May, 1999, observations shown in bold type lie along similar stream lines of flow to that observed using MERLIN on 9 May, 1999, and observations shown in italic type lie along similar stream lines of flow to that observed with MERLIN on 11 May, 1999. The observation shown in italic bold type lies on a stream line between those observed by MERLIN on 9 and 11 May, 1999, but is included as it covers a distance and latitude region otherwise unrepresented in the LASCO/MERLIN/EISCAT dataset

\begin{tabular}{|c|c|c|c|c|c|c|c|c|}
\hline Date & Source & Latitude & Longitude & Distance & Baseline $_{\|}$ & Baseline $_{\perp}$ & Velocity (slow) & $\Delta$ Velocity (slow) \\
\hline & $0321+123$ & -38 & 279 & 42.6 & 210 & 1 & 338 & 16 \\
\hline 990504 & $0318+164$ & -19 & 244 & 30.8 & 239 & 1 & 319 & 18 \\
\hline \multirow[t]{2}{*}{990505} & $0318+164$ & -21 & 228 & 27.2 & 250 & 0 & 345 & 38 \\
\hline & $0431+206$ & -10 & 263 & 89.4 & 233 & 1 & 339 & 42 \\
\hline 990506 & $0318+164$ & -23 & 214 & 23.9 & 255 & 1 & 367 & 117 \\
\hline \multirow[t]{2}{*}{990507} & $0321+123$ & -57 & 206 & 28.7 & 273 & 6 & 384 & 67 \\
\hline & $0431+206$ & -10 & 233 & 82.8 & 239 & 0 & 295 & 128 \\
\hline 990508 & $0321+123$ & -63 & 193 & 26.7 & 243 & 1 & 306 & 171 \\
\hline 990509 & $0321+123$ & -70 & 271 & 24.9 & 223 & 5 & 287 & 150 \\
\hline 990511 & $0433+053$ & -48 & 193 & 85.9 & 221 & 1 & 295 & 69 \\
\hline 990514 & $0433+053$ & -53 & 152 & 79.1 & 193 & 1 & 254 & 85 \\
\hline
\end{tabular}

The large error bars in the velocities determined from observations of $0431+206$ at large distances from the Sun are caused by the low scintillation power received when the ray-path to the source does not pass close to the Sun. The large uncertainties in velocities determined from observations from inside $28 \mathrm{R}$ may arise from real differences in flow speeds in adjacent streams of slow wind, or may be a result of uncertainties in the modelling process as the ray-path approaches strong scattering. Further analysis is necessary before the contributions of these sources of uncertainty can be separated

degree of variation, so that this difference does not imply inconsistency between the studies. The slow solar wind is known to be highly variable in density and velocity so it would not be surprising if different streams had slightly different acceleration profiles too.

\section{Conclusions}

The results of these simultaneous overlapping optical and IPS observations of the slow wind are most satisfactory. There is good agreement between the velocities observed in the same regions by LASCO,

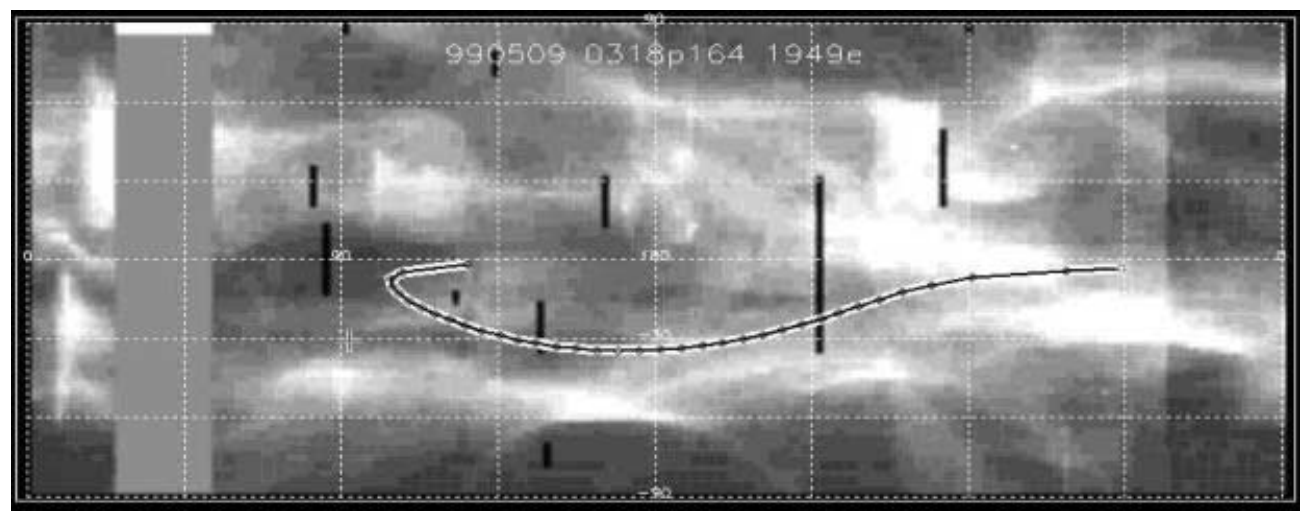

Fig. 4. Ray-path for the MERLIN observation of $0318+164$ on 9 May, 1999, projected back ballistically to $2.5 \mathrm{R}$ and overlaid on a white-light map constructed from east-limb LASCO C2 data. Only a very small portion of the ray-path lies above a coronal hole, which is consistent with the observation being dominated by slow flow. The white-light map covers Carrington longitudes from $-180^{\circ}$ to $180^{\circ}$ and heliographic latitudes from $90^{\circ}$ South to $90^{\circ}$ North, with the centre of the map at $0^{\circ}$ heliographic latitude and $0^{\circ}$ Carrington longitude 


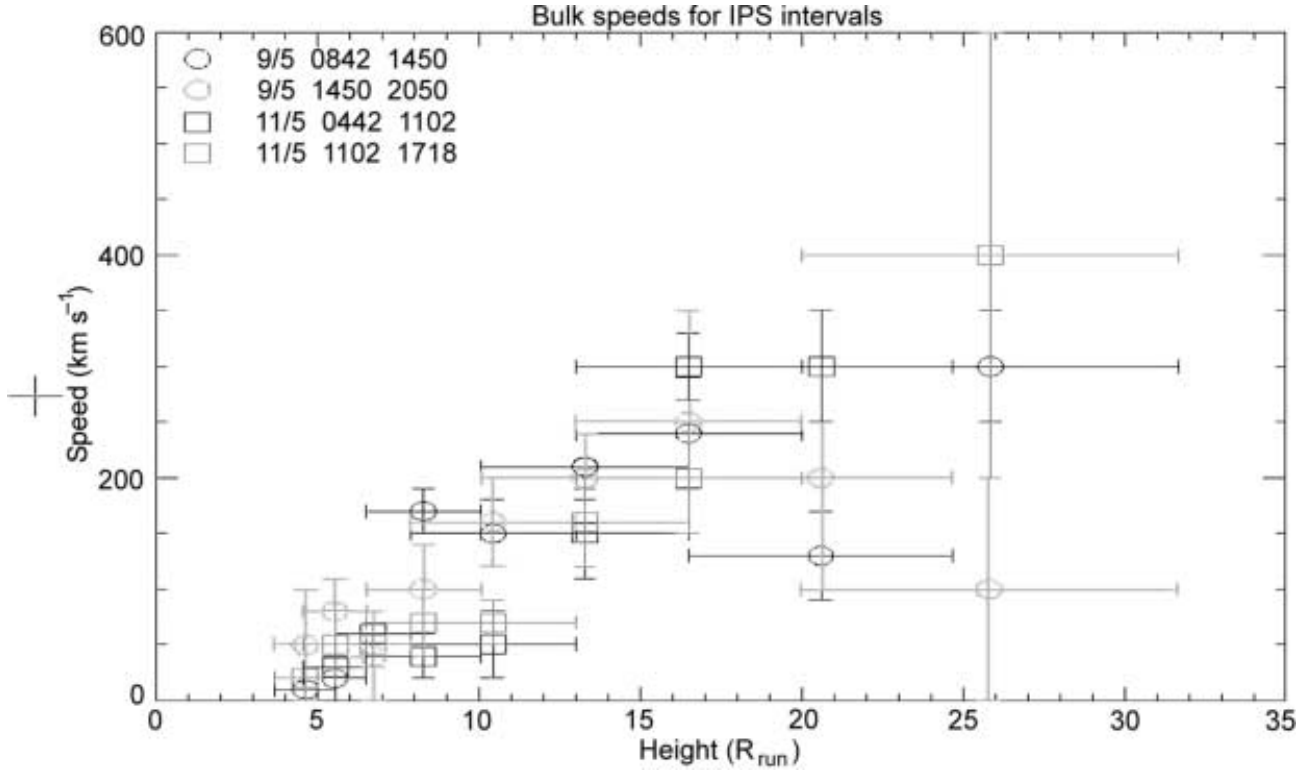

Fig. 5. Slow wind velocities measured by LASCO on 9 and 11 May, 1999. The large error bars for measurements outside $25 \mathrm{R}$ are a result of the low signal-to-noise ratio in the outermost regions of the LASCO field-of-view

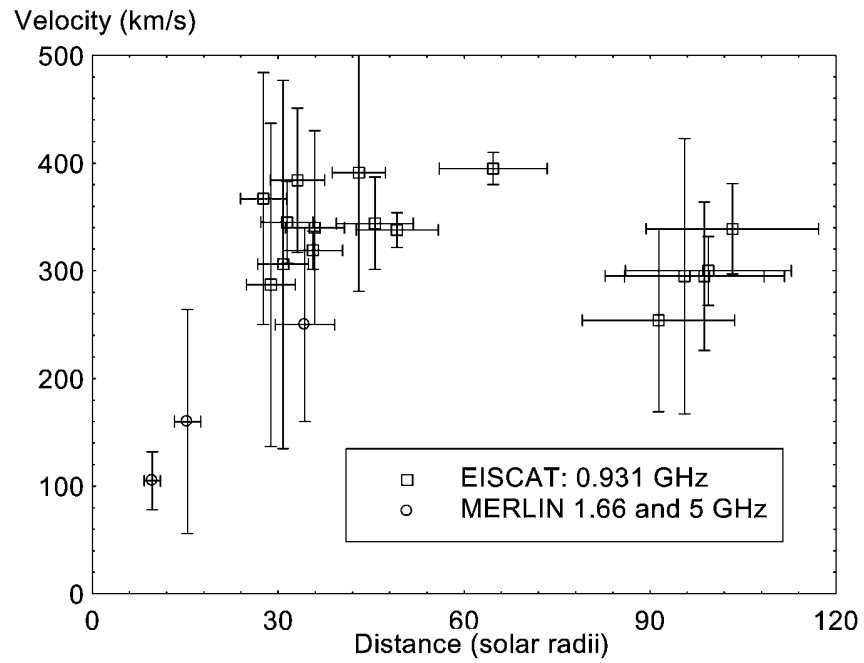

Fig. 6. Velocities determined from EISCAT $\square$ and MERLIN $\bigcirc$ IPS observations off the southeast limb of the Sun between 2 and 15 May, 1999

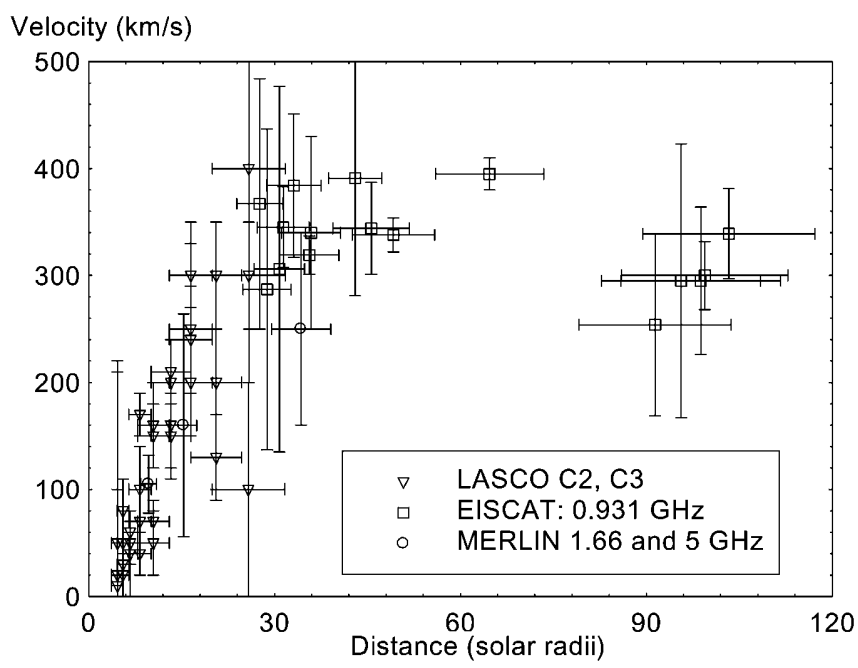

Fig. 7. Combined plot showing LASCO and IPS velocities off the southeast limb of the Sun between 2 and 15 May, 1999
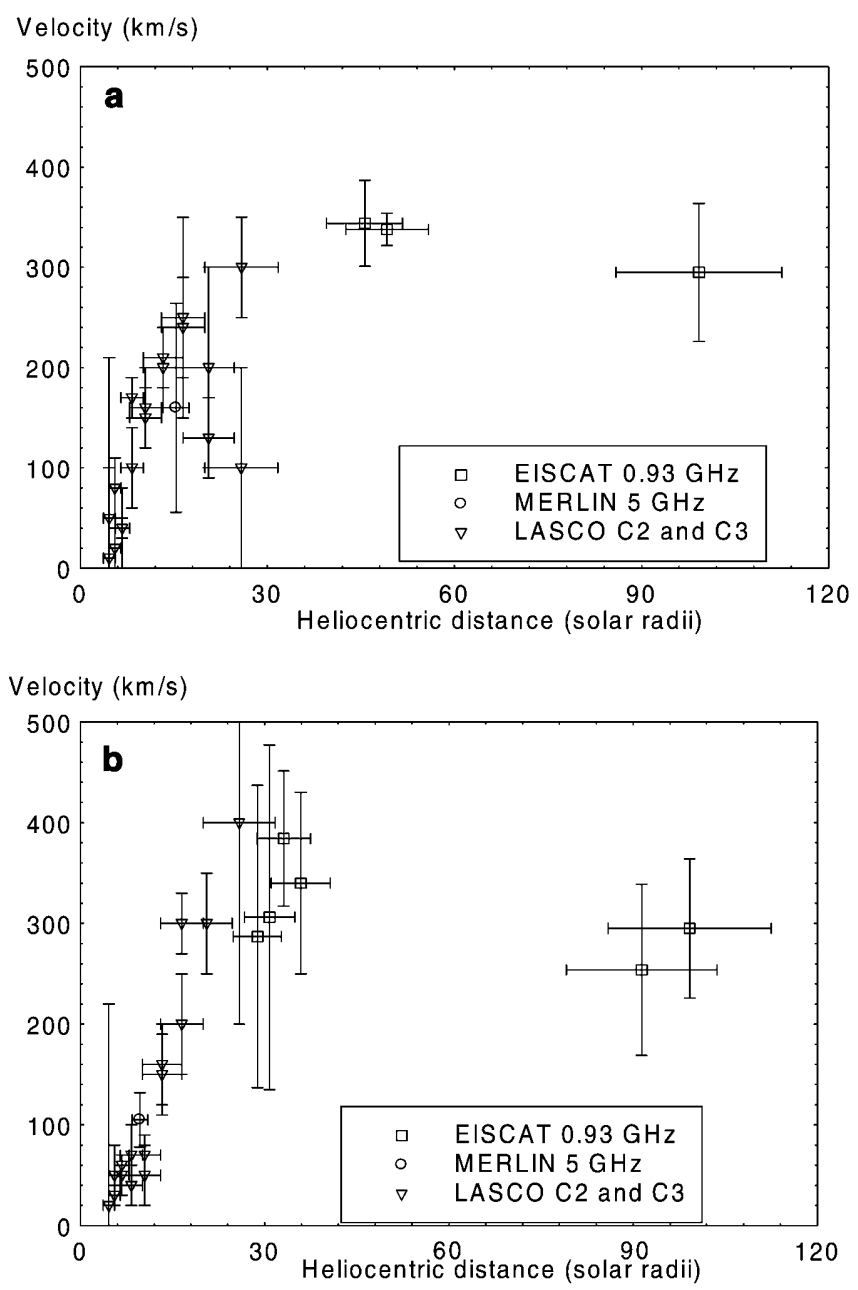

Fig. 8. a Solar wind velocities measured by LASCO, MERLIN and EISCAT along the stream lines of flow observed by LASCO and MERLIN on 9 May, 1999. b As Figure 8a but showing velocities measured by LASCO, MERLIN and EISCAT along the stream lines observed by LASCO and MERLIN on 11 May, 1999 
MERLIN and EISCAT, considering scale sizes of between $10000 \mathrm{~km}$ and $100 \mathrm{~km}$. Together with the similar results of Sheeley et al. (1997) considering "knots" and "blobs" of plasma with scale sizes of $\sim 100000 \mathrm{~km}$, and of Tappin and Simnett (1998) and Srivastava et al. (1999) considering slow-rising CMEs with larger scale sizes, this strongly suggests that density irregularities of very different scale sizes drift at the same speed. This, in turn, supports the Sheeley et al. (1997) picture of density irregularities drifting with the background plasma flow "like leaves in a stream".

The observations made in May 1999 suggest that the slow solar wind begins to accelerate somewhat inside of 4-5 R and reaches its cruising speed by $20-30 \mathrm{R}$, with most acceleration taking place between 10 and $20 \mathrm{R}$. This is very different from the fast wind, which reaches its cruising velocity inside $15 \mathrm{R}$ (Grall et al., 1996; Breen et al., 2000). There is considerable scatter in the measured velocities, and this may reflect differences in speed between adjacent streams of slow wind. The slow wind is known to be highly variable in velocity and density outside 30 R (e.g. Breen et al., 1996b; Axford and McKenzie, 1997) and we would expect these variations to be even more marked close to the Sun, when streamstream interaction will not have had any significant smoothing effect. The variations seen between observations made on 9 and 11 May, 1999, at $36 \pm 10^{\circ}$ and $60 \pm 10^{\circ}$ south latitudes tend to support this view.

The uncertainties in velocities estimated from IPS measurements reflect variation in flow speed across the ray-path. The large uncertainties in measurements made close to the Sun suggest that inside $25 \mathrm{R}$ there is considerable variation in slow wind speed. The very slow lower velocity limits and low coefficients of crosscorrelation seen in the innermost MERLIN observations suggest that quasi-static plasma may be present in the ray-path, coronal streamers are known from LASCO images (Fig. 2) to extend out beyond $8 \mathrm{R}$, so it is possible that very low-speed flow in the body of the streamer may contribute to the wide variation in flow speeds. More observations will be required to confirm or deny this supposition, and we intend to carry out a further sequence of co-ordinated LASCO-MERLINEISCAT measurements in May 2000.

The very good agreement between the drift velocities observed for irregularities of a wide range of scale sizes is an important result. The observations do not explicitly confirm that the irregularity drift velocity is the same as the background flow speed (it is unlikely that anything other than in-situ measurements could unambiguously measure the bulk flow speed in this region), but the agreement seen between the drift velocities of irregularities of such different scale sizes does suggest that these features are indeed drifting with the background flow. The slow and variable nature of the acceleration profile observed is very different to that seen in the fast wind, and will provide valuable information for modellers seeking to understand the origin and acceleration of the slow solar wind.

A small amount of the ray-paths of all of these observations above dark regions in the white-light maps, and LASCO, MERLIN and EISCAT all detected small proportions of fast flow. More analysis is required to accurately determine the precise velocities in these regions, and we hope to present these results in a future paper.

Acknowledgements. We would like to thank the director and staff of EISCAT for the $0.93 \mathrm{GHz}$ IPS data used in this study. EISCAT is supported by the scientific research councils of Finland, France, Germany, Japan, Norway, Sweden and the U.K. MERLIN is a National Facility operated by the University of Manchester on behalf of PPARC. We would like to thank the Director and staff of Jodrell Bank for making available necessary additional equipment. The LASCO white-light data was made available by the LASCO consortium. SOHO is an ESA/NASA joint mission. We would like to give particular thanks to B.J. Rickett and W.A. Coles for making their experimental programmes, analysis routines and expertise available to us. Three of us (ARB, RAF, PJM) were supported by PPARC during the period when this work was carried out. AC was supported by the University of Wales.

Topical Editor M. Lester thanks R. Schwenn and G. Woan for their help in evaluating this paper.

\section{References}

Armstrong, J. A., and W. A. Coles, Analysis of three-station interplanetary scintillation data, J. Geophys. Res., 77, 46024610, 1972.

Axford, W. I., and J. F. McKenzie, The solar wind, in Cosmic winds and the heliosphere, Eds. Jokipii, J. R., C. P. Sonnett, and M. S. Giampapa, University of Arizona Press, 1997.

Bourgois, G., W. A. Coles, G. Daigne, J. Silen, T. Turenen, and P. J. S. Williams, Measurements of solar wind velocity using EISCAT, Astron. Astrophys., 144, 452-462, 1985.

Breen, A. R., W. A. Coles, R. R, Grall, U. P. Lovhaug, J. Markkanen, H. Misawa, and P. J. S. Williams, EISCAT observations of interplanetary scintillation, J. Atmos. Terr. Phys., 58, 507-519, 1996a.

Breen, A. R., W. A. Coles, R. R. Grall, M. T. Klinglesmith, J. Markkanen, P. J. Moran, B. Tegid, and P. J. S. Williams, EISCAT measurements of the solar wind, Ann. Geophysicae, 14, 1235-1245, 1996b.

Breen, A. R., C. F. De Forest, B. J. Thompson, J. F. McKenzie, A. Modigliani, P. J. Moran, C. A. Varley, and P. J. S. Williams, Comparisons of interplanetary scintillation and optical measurements of solar wind acceleration with model results, $A d v$. Space Res. in press, 2000.

Brueckner, G. E. et al., The large angle spectroscopic coronagraph (LASCO), Sol. Phys., 162, 357-402, 1995.

Coles, W. A., Interplanetary scintillation observations of the highlatitude solar wind, Space Sci. Rev., 72, 211-222, 1995.

Dennison, P. A., and A. Hewish, The solar wind outside the plane of the ecliptic, Nature, 213, 343-346, 1967.

Domingo, V., B. Fleck, and A. I. Poland, The SOHO mission: an overview, Sol. Phys., 162, 1-37, 1995.

Grall, R. R., Remote sensing observations of the solar wind near the Sun, PhD Thesis, University of California, San Diego, 1995.

Grall, R. R., M. T. Klinglesmith, W. A. Coles, A. R. Breen, P. J. S. Williams, and R. Esser, Rapid acceleration of the polar solar wind, Nature, 379, 429-432, 1996.

Hewish, A., A user's guide to scintillation, J. Atmos. Terr. Phys., 51, 743-750, 1989.

Hewish, A., P. F. Scott, and D. Willis, Interplanetary scintillation of small-diameter radio sources, Nature, 203, 1214-1217, 1964.

Moran, P. J., A. R. Breen, C. A. Varley, P. J. S. Williams, W. P. Wilkinson, W. A. Coles, and J. Markkanen, Measurements of the direction of the solar wind using interplanetary scintillation, Ann. Geophysicae, 16, 1259-1264, 1998. 
Rickett, B., IPS observations of the solar wind velocity and microscale density irregularities in the inner solar wind, in Solar Wind 7, Proceedings of the 3rd COSPAR Colloqium held in Goslar, Germany, 16-20 September 1991, COSPAR Colloquia Series, vol 3, E. Marsch and R. Schwenn, Eds., Pergamon, 255258, 1992.

Sheeley, N. R. et al., Measurements of flow speeds in the corona between 2 and 30 R, Astrophys. J., 484, 472-478, 1997.

Srivastava N, R. Schwenn, B. Inhester, G. Stenborg, and B. Podlipnik, Acceleration profile of the slow solar wind as inferred from gradual mass ejections observed by LASCO, Space Sci. Rev., 87, 303-306, 1999.

Tappin, S. J., and G. M. Simnett, The acceleration of CMEs in the outer corona as revealed by LASCO, in Proc. 31st ESLAB Symposium, ESA-SP-415, Ed. A. Wilson, 117-121, 1998.

Tappin, S. J., G. M. Simnett, and M. A. Lyons, A determination of the outflow speeds in the lower solar wind, Astron. Astrophys., 350, 302-309, 1999.

Thomasson, P., MERLIN, Q. J. R. Astr. Soc., 27, 413-431, 1986. 